\title{
Regular Ultrapowers at Regular Cardinals
}

\author{
Juliette Kennedy, Saharon Shelah, and Jouko Väänänen
}

\begin{abstract}
In earlier work by the first and second authors, the equivalence of a finite square principle $\square_{\lambda, D}^{\text {fin }}$ with various model-theoretic properties of structures of size $\lambda$ and regular ultrafilters was established. In this paper we investigate the principle $\square_{\lambda, D}^{\mathrm{fin}}$-and thereby the above model-theoretic properties—at a regular cardinal. By Chang's two-cardinal theorem, $\square_{\lambda, D}^{\text {fin }}$ holds at regular cardinals for all regular filters $D$ if we assume the generalized continuum hypothesis $(\mathrm{GCH})$. In this paper we prove in ZFC that, for certain regular filters that we call doubly ${ }^{+}$ regular, $\square \square_{\lambda, D}^{\text {fin }}$ holds at regular cardinals, with no assumption about GCH. Thus we get new positive answers in ZFC to Open Problems 18 and 19 in Chang and Keisler's book Model Theory.
\end{abstract}

\section{Introduction}

In Kennedy and Shelah [7], [8] the equivalence of the following finite square principle $\square_{\lambda, D}^{\text {fin }}$ with various model-theoretic properties of regular reduced powers of models was established:

$\square_{\lambda, D}^{\text {fin }}: D$ is a filter on a cardinal $\lambda$, and there exist finite sets $C_{\alpha}^{\xi}$ and integers $n_{\xi}$ for each $\alpha<\lambda^{+}$and $\xi<\lambda$ such that for each $\xi, \alpha$,

(i) $C_{\alpha}^{\xi} \subseteq \alpha+1$;

(ii) if $B \subset \lambda^{+}$is a finite set of ordinals and $\alpha<\lambda^{+}$is such that $B \subseteq \alpha+1$, then $\left\{\xi: B \subseteq C_{\alpha}^{\xi}\right\} \in D$;

(iii) $\beta \in C_{\alpha}^{\xi}$ implies $C_{\beta}^{\xi}=C_{\alpha}^{\xi} \cap(\beta+1)$;

(iv) $\left|C_{\alpha}^{\xi}\right|<n_{\xi}$.

The model-theoretic properties were the following. First, if $D$ is an ultrafilter, then $\square_{\lambda, D}^{\text {fin }}$ is equivalent to $\mathcal{M}^{\lambda} / D$ being $\lambda^{++}$-universal for each model $\mathcal{M}$ in a vocabulary of size at most $\lambda$. To formulate the second model-theoretic property, let us

Received July 18, 2012; accepted February 7, 2013

2010 Mathematics Subject Classification: Primary 03C20 ; Secondary 03 E05

Keywords: regular filter, square principle, good ultrafilter, ultraproduct, reduced product (C) 2015 by University of Notre Dame 10.1215/00294527-3132788 
say that two models are $E F_{\alpha}$-equivalent if the second player (i.e., the isomorphism player) has a winning strategy in the Ehrenfeucht-Fraïssé game of length $\alpha$ on the two models. ${ }^{1}$ Now $\square_{\lambda, D}^{\text {fin }}$ is equivalent to $\mathcal{M}^{\lambda} / D$ and $\mathcal{N}^{\lambda} / D$ being $E F_{\lambda+\text {-equivalent }}$ for any elementarily equivalent models $\mathcal{M}$ and $\mathcal{N}$ (without loss of generality of cardinality at most $\lambda^{+}$) in a vocabulary of size at most $\lambda$. The existence of such ultrafilters and models is related to Open Problems 18 and 19 in Chang and Keisler's book on model theory [1].

The consistency of the failure of $\square_{\lambda, D}^{\text {fin }}$ for a regular filter at a singular strong limit cardinal $\lambda$ was proved in [8, Corollary 6] relative to the consistency of a supercompact cardinal. In Kennedy, Shelah, and Väänänen [9, Corollary 8] this was improved to the failure of $\square_{\lambda, D}^{\text {fin }}$ for a regular (ultra)filter $D$ at a singular strong limit cardinal $\lambda$ relative to the consistency of a strongly compact cardinal. The failure of $\square_{\lambda, D}^{\text {fin }}$ for an ultrafilter implies the failure of $\lambda^{++}$-universality of $\mathcal{M}^{\lambda} / D$ for some $\mathcal{M}$, as well as the failure of isomorphism of some regular ultrapowers $\mathcal{M}^{\lambda} / D$ and $\mathcal{N}^{\lambda} / D$. Thus [9] answered negatively the following problems listed in [1] modulo large cardinal assumptions.

Problem 18 ([1]) Let $|M|,|N|,|L| \leq \alpha$, and let $D$ be a regular ultrafilter over $\alpha$. If $\mathcal{M} \equiv \mathcal{N}$, then $\prod_{D} \mathcal{M} \cong \prod_{D} \mathcal{N}$.

Problem 19 ([1]) If $D$ is a regular ultrafilter of $\alpha$, then for all infinite $\mathcal{M}, \prod_{D} \mathcal{M}$ is $\alpha^{++}$-universal.

The use of large cardinals is justified by [7], [8], and [12] as the failure of $\square_{\lambda, D}^{\text {fin }}$, for a singular strong limit $\lambda$ implies the failure of $\square_{\lambda}$, which implies the consistency of large cardinals.

In this paper we investigate the principle $\square_{\lambda, D}^{\text {fin }}$, and thereby the above modeltheoretic problems, at a regular cardinal. The following result is proved in Hyttinen [4, Theorem 3.3]. Assume that $\kappa$ is regular and that $\lambda^{<\kappa}=\lambda$. Suppose that $\mathcal{M}$ and $\mathcal{N}$ are structures for a finite vocabulary such that $\mathcal{M}$ and $\mathcal{N}$ are $E F_{\alpha}$-equivalent for each $\alpha<\kappa$. Suppose that $D$ is a filter on $\xi \times \lambda, \xi \leq \lambda$, extending $F^{\prime} \times F$, where $F^{\prime}$ is a $\kappa$-descendingly incomplete filter on $\xi$ and $F$ is a $\kappa$-semigood filter on $\lambda$ (the concept is defined in [4, Definition 3.2]). Then $\mathcal{M}^{\lambda} / D$ and $\mathcal{N}^{\lambda} / D$ are $E F_{\lambda+}$-equivalent. For $\kappa=\omega$ this, combined with the existence proof of semigood filters in [4, Lemma 3.5], yields filters $D$ with $\square_{\lambda, D}^{\text {fin }}$. The structure of the paper is the following. In Section 2 we prove weaker versions of $\square_{\lambda, D}^{\mathrm{fin}}$ in the case where the filter $D$ extends the club filter on $\lambda$. Naturally this case is in spirit quite far from the case of regular $D$, which is our prime interest. However, this result is useful in the sequel. Note that there are many regular (ultra)filters extending the club filter. In Section 3 we define the concept of doubly ${ }^{+}$regular filter and show that such filters $D$ on regular $\lambda>\aleph_{0}$ satisfy $\square_{\lambda, D}^{\text {fin }}$. Thus we get new positive answers in $Z F C$ to the above Problem 18 (with isomorphism replaced, in the absence of $2^{\lambda}=\lambda^{+}$,

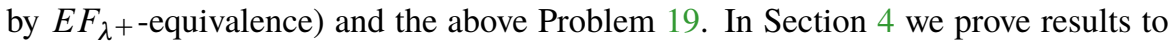
the effect that not all regular filters are doubly regular. In Section 5 we compare our concept of double regularity to Keisler's concept of goodness of a filter. In Section 6 we present some open questions. 


\section{Filters Extending the Club Filter}

We can get provable cases of a weaker form of $\square_{\lambda, D}^{\text {fin }}$, when $D$ extends the club filter. This will prove useful in the next section, where we will use Theorem 1 in the proof of Theorem 5. The original $\square_{\lambda, D}^{\mathrm{fin}}$ is equivalent to reduced powers of elementarily equivalent models of cardinality $\lambda$ being $E F_{\lambda+}$-equivalent. The weaker form (which we prove below) will give the $E F_{\lambda+}$-equivalence of reduced powers of models of power $\lambda$ that are not just elementarily equivalent but even $E F_{\lambda}$-equivalent.

\section{Theorem 1 Suppose that}

(a) $\lambda$ is regular $>\boldsymbol{\aleph}_{0}$,

(b) $D$ is a filter on $\lambda$,

(c) D extends the club filter.

If $\mathcal{M}$ and $\mathcal{N}$ are $E F_{\lambda}$-equivalent, then $\mathcal{M}^{\lambda} / D$ and $\mathcal{N}^{\lambda} / D$ are $E F_{\lambda+- \text {-equivalent. }}$

Proof If $\alpha<\lambda^{+}, \lambda$ regular, let $\left\{u_{\alpha}^{i}: i<\lambda\right\}$ be a continuously increasing sequence of subsets of $\alpha$ such that $\left|u_{\alpha}^{i}\right|<\lambda$ for all $i<\lambda$ and $\alpha=\bigcup_{i<\lambda} u_{\alpha}^{i}$. Let

$$
D_{\alpha}=\left\{i<\lambda: \forall \beta \in u_{\alpha}^{i}\left(u_{\beta}^{i}=u_{\alpha}^{i} \cap \beta\right)\right\} .
$$

It is easy to see that $D_{\alpha}$ is a club of $\lambda$ (recall that $\lambda$ is regular).

Now we can proceed, as in [7], to prove that if $M$ and $N$ are $E F_{\lambda}$-equivalent, then $M^{\lambda} / D$ and $N^{\lambda} / D$ are $E F_{\lambda+}$-equivalent.

Let $L$ be a finite vocabulary, and for each $i<\lambda$, let $\mathcal{M}_{i}$ and $\mathcal{N}_{i}$ be $E F_{\lambda}$-equivalent $L$-structures. We show that II has a winning strategy in the game $E F_{\lambda+}$ on the models $\mathcal{M}=\prod_{D} \mathcal{M}_{i}$ and $\mathcal{N}=\prod_{D} \mathcal{N}_{i}$.

The crucial idea of the proof is the following: When the Ehrenfeucht-Fraïssé game $E F_{\lambda+}(\mathcal{M}, \mathcal{N})$ is played, the players are actually playing $\lambda$ Ehrenfeucht-Fraïssé games simultaneously, namely, the games $E F_{\lambda}\left(\mathcal{M}_{i}, \mathcal{N}_{i}\right), i<\lambda$.

For each $i<\lambda$, let $\sigma_{i}$ be a winning strategy for II in the game $E F_{\lambda}$ on the models $\mathcal{M}_{i}$ and $\mathcal{N}_{i}$. A good position is a sequence $\left\langle\left(f_{\beta}, g_{\beta}\right): \beta<\alpha\right\rangle$ for some $\alpha<\lambda^{+}$, together with a club $C \subseteq D_{\alpha}$, such that for all $\beta<\alpha$ we have $f_{\beta} \in \prod_{i} M_{i}$, $g_{\beta} \in \prod_{i} N_{i}$, and if $i \in C$, then

$$
\left\langle\left(f_{\eta}(i), g_{\eta}(i)\right): \eta \in u_{\alpha}^{i}\right\rangle
$$

is a play according to $\sigma_{i}$ on the models $\mathcal{M}_{i}$ and $\mathcal{N}_{i}$. In a good position the equivalence classes of the functions $f_{\beta}$ and $g_{\beta}$ determine a partial isomorphism of the reduced products. Suppose that $\alpha$ rounds have been played and that we are in a good position. Let $\varphi_{\gamma}\left(\left[f_{\beta_{1}}\right], \ldots,\left[f_{\beta_{k}}\right]\right)$ be an atomic formula holding in $\prod_{i} \mathcal{M}_{i} / D$, where $\beta_{1}<\cdots<\beta_{k}<\alpha$, and let $A=\left\{i \in D_{\alpha}:\left\{\beta_{1}, \ldots, \beta_{k}\right\} \subseteq u_{\alpha}^{i}\right\}$. By assumption, $A \in D$. Since also $B=\left\{i<\lambda: \mathcal{M}_{i} \models \varphi_{\gamma}\left(f_{\beta_{1}}(i), \ldots, f_{\beta_{k}}(i)\right)\right\} \in D$, we have $A \cap B \in D$. For $i \in A \cap B$, we have $\beta_{1}, \ldots, \beta_{k} \in u_{\alpha}^{i}$; hence

$$
u_{\beta_{j}}^{i}=u_{\alpha}^{i} \cap \beta_{j} \text {. }
$$

Since we are in a good position, $\left\langle\left(f_{\eta}(i), g_{\eta}(i)\right): \eta \in u_{\alpha}^{i}\right\rangle$ is a play according to winning strategy $\sigma_{i}$. Hence $\left\langle\left(f_{\epsilon}(\xi), g_{\epsilon}(\xi)\right): \epsilon \in u_{\alpha}^{i}\right\rangle$ determines a partial isomorphism of the structures $\mathcal{M}_{i}$ and $\mathcal{N}_{i}$. Since this was the case for all $i \in A \cap B \in D$, we get $\prod_{\epsilon} \mathcal{N}_{\epsilon} / D \models \varphi_{\gamma}\left(\left[g_{\beta_{1}}\right], \ldots,\left[g_{\beta_{k}}\right]\right)$. 
The strategy of II is to keep the position of the game good and thereby win the game. So suppose $\beta$ rounds have been played and II has been able to keep the position good. Then for all $\gamma<\beta$ there is a club $C_{\gamma} \subseteq D_{\gamma}$ such that for $i \in C_{\gamma}$, $\left\langle\left(f_{\eta}(i), g_{\eta}(i)\right): \eta \in u_{\gamma}^{i}\right\rangle$ is a play according to $\sigma_{i}$.

Case 1: $\beta=\bigcup \beta$. Let $C=\bigcap_{\gamma<\beta} C_{\gamma}$. Since $\lambda$ is regular, this is still a club. We show that $\left\langle\left(f_{\gamma}, g_{\gamma}\right): \gamma<\beta\right\rangle$ is good. Let $i \in C$. Let us look at $\left\langle\left(f_{\eta}(i), g_{\eta}(i)\right): \eta \in u_{\beta}^{i}\right\rangle$. Since $i \in D_{\beta}$, every initial segment of this play is a play according to $\sigma_{i}$. Hence so is the entire play $\left\langle\left(f_{\gamma}, g_{\gamma}\right): \gamma<\beta\right\rangle$. We have shown that II can maintain a good position.

Case 2: $\beta=\delta+1$. Let $C \subseteq \bigcap_{\gamma \leq \beta} C_{\gamma}$ such that $\delta \in u_{\beta}^{i}$ for $i \in C$. Now suppose I plays $f_{\delta}$. We show that II can play $g_{\delta}$ so that $\left\langle\left(f_{\gamma}, g_{\gamma}\right): \gamma<\beta\right\rangle$ remains good. Let $i \in C$. Let us look at $\left\langle\left(f_{\eta}(i), g_{\eta}(i)\right): \eta \in u_{\delta}^{i}\right\rangle$. This is a play according to the strategy $\sigma_{i}$. Since $i \in D_{\beta}$ and $\delta \in u_{\beta}^{i}, u_{\delta}^{i}=u_{\beta}^{i} \cap \delta$, so after the moves $\left\langle\left(f_{\eta}(i), g_{\eta}(i)\right): \eta \in u_{\delta}^{i}\right\rangle$ II can play one more move in $E F_{\lambda}$ on $\mathcal{M}_{i}$ and $\mathcal{N}_{i}$ with I playing the element $f_{\delta}(i)$. Let $g_{\delta}(i)$ be the answer of II in this game according to $\sigma_{i}$. The values $g_{\delta}(i), i \in C$, constitute the function $g_{\delta} \bmod D$. We have shown that II can maintain a good position.

We do not know whether the conditions (a)-(c) of Theorem 1 are necessary for the conclusion.

Remark 2 We point out some variants of Theorem 1.

1. We can define a version $\square_{\lambda, D}^{\gamma}$ of $\square_{\lambda, D}^{\text {fin }}$ which is equivalent to: "If $\mathcal{M}$ and $\mathcal{N}$ are $E F_{\gamma}$-equivalent, then $\mathcal{M}^{\lambda} / D$ and $\mathcal{N}^{\lambda} / D$ are $E F_{\lambda+\text {-equivalent": }}$

$\square_{\lambda, D}^{\gamma}: D$ is a filter on a cardinal $\lambda$, and there exist finite sets $C_{\alpha}^{\xi}$ and ordinals $\gamma_{\xi}<\gamma$ for each $\alpha<\lambda^{+}$and $\xi<\lambda$ such that for each $\xi, \alpha$

(i) $C_{\alpha}^{\xi} \subseteq \alpha+1$;

(ii) if $B \subset \lambda^{+}$is a set of ordinals with $\operatorname{otp}(B)<\gamma$ and $\alpha<\lambda^{+}$is such that $B \subseteq \alpha+1$, then $\left\{\xi: B \subseteq C_{\alpha}^{\xi}\right\} \in D$;

(iii) $\beta \in C_{\alpha}^{\xi}$ implies $C_{\beta}^{\xi}=C_{\alpha}^{\xi} \cap(\beta+1)$;

(iv) $\operatorname{otp}\left(C_{\alpha}^{\xi}\right)<\gamma_{\xi}$.

If clauses (a), (b), and (c) of Theorem 1 are assumed, then $\square_{\lambda, D}^{\lambda}$.

2. We can also define a version $\square_{\lambda, D}^{<\delta}$ of $\square_{\lambda, D}^{\text {fin }}$ which is equivalent to: "If $\mathcal{M}$ and $\mathcal{N}$ are $E F_{\gamma}$-equivalent for all $\gamma<\delta$, then $\mathcal{M}^{\lambda} / D$ and $\mathcal{N}^{\lambda} / D$ are $E F_{\lambda+}$-equivalent." If clauses (a), (b), and (c) ${ }^{+}$of Theorem 1 are assumed, then $\square_{\lambda, D}^{<\lambda}$ holds, where $(c)^{+}$says that (c) holds and there are functions $f_{\alpha}$, $\alpha \leq \lambda^{+}$, such that $\alpha<\beta \leq \lambda^{+}$implies $\left\{i<\lambda: f_{\alpha}(i)<f_{\beta}(i)\right\} \in D$. (For $D=$ the club filter, this is the so-called assumption of the existence of the $\lambda^{+}$th canonical function; see, e.g., Jech [5, p. 445].)

3. Note that for $\gamma<\lambda$.

$$
\square_{\lambda, D}^{\mathrm{fin}} \Rightarrow \square_{\lambda, D}^{\gamma} \Rightarrow \square_{\lambda, D}^{<\lambda} \Rightarrow \square_{\lambda, D}^{\lambda}
$$

4. We get a variant of Theorem 1 also by showing, assuming (a), (b), and (c), that $\prod_{D} \mathcal{M}_{i}$ and $\prod_{D} \mathcal{N}_{i}$ are $E F_{\lambda+}$-equivalent, if for all $\beta<\lambda$ :

$\left\{i<\lambda: \mathcal{M}_{i}\right.$ and $\mathcal{N}_{i}$ are $E F_{\beta}$-equivalent $\} \in D$. 
5. We can weaken clause (c) of the theorem to the assumption that $D$ is unreasonable (see Shelah [14]) in the following sense: There is a partition $\left\{w_{i}: i<\lambda\right\}$ of $\lambda$ such that $\bigcup_{i \in E} w_{i} \in D$ for every club $E$ of $\lambda$.

\section{Doubly Regular Filters}

We define the concept of a doubly regular filter, give examples of such on regular cardinals, and prove that $\square_{\lambda, D}^{\text {fin }}$ holds for such filters. Recall that a family of sets is a regular family if finite intersections of members of the family are nonempty, but all infinite intersections are empty, a filter is called $\mu$-regular if it contains a regular family of size $\mu$, and a filter on $\lambda$ is called regular if it is $\lambda$-regular.

Definition 3 Suppose that $D$ is a filter on a regular cardinal $\lambda$.

1. $D$ is called doubly regular if there are pairwise disjoint sets $u_{i} \subseteq \lambda, i<\lambda$, each of cardinality $\lambda$, and regular filters $D_{i}$ on $u_{i}$ such that for all $A \subseteq \lambda$ :

$$
\left[\forall^{\infty} i<\lambda\left(A \cap u_{i} \in D_{i}\right)\right] \Rightarrow A \in D
$$

(" $\forall \infty_{i}<\lambda$ " means "for all but boundedly many $i$ ").

2. The filter $D$ is called doubly ${ }^{+}$regular if the above holds with " $\forall \infty_{i}<\lambda$ " replaced by "for a club of $i$."

Let us make some easy observations about doubly regular filters.

\section{Observation 4}

1. A doubly regular filter is necessarily regular. Let $\left\{A_{i}^{\alpha}: \alpha<\lambda\right\}$ be a regular family in $D_{i}$. Let

$$
B^{\alpha}=\bigcup_{i<\lambda} A_{i}^{\alpha}
$$

Then $\left\{B^{\alpha}: \alpha<\lambda\right\}$ is a regular family in D. We will show in Theorem 7 below that the converse need not be true.

2. A doubly ${ }^{+}$regular filter is always doubly regular.

3. It is easy to construct doubly $\left({ }^{+}\right)$regular filters. Indeed, if the sets $u_{i} \subseteq \lambda$, $i<\lambda$, are disjoint, each of cardinality $\lambda, \lambda=\bigcup_{i} u_{i}$, and we have regular filters $D_{i}$ on $u_{i}$, then the set $\left\{A \subseteq \lambda: \forall^{\infty} i<\lambda\left(A \cap u_{i} \in D_{i}\right)\right\}$ is a doubly regular filter on $\lambda$, and the larger set $\left\{A \subseteq \lambda\right.$ : for a club of $\left.i<\lambda\left(A \cap u_{i} \in D_{i}\right)\right\}$ is a doubly ${ }^{+}$regular filter on $\lambda$. Both double regularity and double ${ }^{+}$regularity are closed under extensions of the filter, so we get also ultrafilter examples of both.

Here is the main point of doubly ${ }^{+}$regular filters, at least from the point of view of this paper.

Theorem 5 If $D$ is a doubly ${ }^{+}$regular filter on a regular cardinal $\lambda>\boldsymbol{\aleph}_{0}$, then $\square_{\lambda, D}^{\text {fin }}$ holds.

Proof Let the sets $u_{i}$ and the filters $D_{i}$ be as in Definition 3. Let $D^{*}$ be the club filter of $\lambda$, and let

$$
D^{\prime}=\left\{A \subseteq \lambda:\left\{i<\lambda: A \cap u_{i} \in D_{i}\right\} \in D^{*}\right\} .
$$

We prove $\square_{\lambda, D^{\prime}}^{\text {fin }}$. From this $\square_{\lambda, D}^{\text {fin }}$ follows, as $D^{\prime} \subseteq D$. It suffices to prove that if $\mathcal{M}_{\alpha}$ and $\mathcal{N}_{\alpha}, \alpha<\lambda$, are elementarily equivalent, with a vocabulary of size at most $\lambda$, then $\mathcal{M}=\prod_{D^{\prime}} \mathcal{M}_{\alpha}$ and $\mathcal{N}=\prod_{D^{\prime}} \mathcal{N}_{\alpha}$ are $E F_{\lambda+\text {-equivalent. Note that }}$ 
(a) $\mathcal{M} \cong \prod_{i<\lambda} \mathcal{M}^{i} / D^{*}$, where $\mathcal{M}^{i}=\prod_{\alpha \in u_{i}} \mathcal{M}_{\alpha} / D_{i}$;

(b) $\mathcal{N} \cong \prod_{i<\lambda} \mathcal{N}^{i} / D^{*}$, where $\mathcal{N}^{i}=\prod_{\alpha \in u_{i}} \mathcal{N}_{\alpha} / D_{i}$.

Since each $D_{i}$ is $\lambda$-regular, the models $\mathcal{M}^{i}$ and $\mathcal{N}^{i}$ are $E F_{\lambda}$-equivalent by Shelah [13, Theorem VI.1.8]. By Theorem 1 the models $\mathcal{M}$ and $\mathcal{N}$ are now $E F_{\lambda+- \text {-equivalent. }}$

\section{On Regular But Nondoubly Regular Filters}

Nonregular uniform filters do not necessarily exist. If there is a nonregular uniform ultrafilter on $\omega_{1}$, then $V \neq L$ by Prikry [11], $0^{\#}$ exists by Ketonen [10], and in fact $\omega_{2}$ is a limit of measurable cardinals in the Dodd-Jensen core model by Deiser and Donder [2]. We show that we can always construct a regular but nondoubly regular filter. In this sense, double regularity is easier to avoid than regularity.

If $E$ is an equivalence relation on $\lambda$, we denote the set of all $E$-classes by $\lambda / E$, and the $E$-class of $i$ by $i / E$.

First we give an equivalent condition for double regularity, one that better fits our present purpose.

Lemma 6 A filter $D$ is doubly regular if and only if there is an equivalence relation $E$ of $\lambda$ and $\bar{u}=\left\langle u_{\alpha}: \alpha \in \lambda\right\rangle$ such that

(DR-a) $\left\{u_{\epsilon}: \epsilon \sim_{E} i\right\}$ is a regular family of subsets of $i / E$ for each $i<\lambda$;

(DR-b) if $S \subseteq \lambda$ and $|S|<\lambda$, then $\bigcup\{i / E: i \in S\}=\emptyset \bmod D$;

(DR-c) $|i / E|=\lambda$ for all $i<\lambda$;

(DR-d) if $f$ is a function such that $\operatorname{dom}(f)=\lambda / E$ and $f(i / E) \sim_{E} i$ for all $i \in \lambda / E$, then $\bigcup_{i \in \lambda / E} u_{f(i)} \notin D$.

The proof is easy.

Theorem $7 \quad$ If $2^{\lambda}=\lambda^{+}$, then there is a regular ultrafilter on $\lambda$ which is not doubly regular.

Proof Let $\left\{B_{\alpha}: \alpha \in \lambda^{+}\right\}$list $\mathcal{P}(\lambda)$. Let $\left\{\left(E^{\alpha}, \bar{u}_{\alpha}\right): \alpha<\lambda^{+}\right\}$list potential candidates for double regularity; that is, $E$ and $\bar{u}=\left\langle u_{\zeta}: \zeta<\lambda\right\rangle$ such that $\left\{u_{\zeta}: \zeta<i / E\right\}$ is a regular family on $i / E$ for each $i<\lambda$. This is only place where we use $2^{\lambda}=\lambda^{+}$.

We construct by induction sets $\mathscr{D}_{\alpha}, \alpha<\lambda^{+}$, such that the following conditions will hold:

(C-a) $\mathscr{D}_{\alpha} \subseteq \mathcal{P}(\lambda)$ is $\subseteq$-continuously increasing;

(C-b) $\left|\mathscr{D}_{\alpha}\right|=\lambda$;

(C-c) $\mathscr{D}_{\alpha}$ is closed under finite intersections (we use Fil $\left(\mathscr{D}_{\alpha}\right)$ to denote the filter $\mathscr{D}_{\alpha}$ generates);

(C-d) $\mathscr{D}_{0}$ contains a regular family (so necessarily, $u \in[\lambda]^{<\lambda}$ implies $u=\varnothing$ $\bmod D)$

(C-e) if $\alpha=2 \beta+1$, then $B_{\beta} \in \mathscr{D}_{\alpha}$ or $\left(\lambda \backslash B_{\beta}\right) \in \mathscr{D}_{\alpha}$;

(C-f) if $\alpha=2 \beta+2$, then either there is $S \in[\lambda]^{<\lambda}$ such that $\bigcup_{\epsilon \in S} \epsilon / E_{\beta} \neq \emptyset$ $\bmod \operatorname{Fil}\left(\mathscr{D}_{\alpha}\right)$, or, letting $\bar{u}_{\beta}=\left\langle u_{\beta, \epsilon}: \epsilon<\lambda\right\rangle$, there is $f$ such that $\operatorname{dom}(f)=\lambda / E_{\beta}, f\left(i / E_{\beta}\right) \sim_{E_{\beta}} i$ for all $i \in \lambda / E_{\beta}$, and $\bigcup_{i \in \lambda / E_{\beta}} u_{\beta, f(i)} \in D_{a}$.

Here is the construction. 
Case 1: $\alpha=0$. Let $E$ be a regular family on $\lambda$. (We can construct a regular family on $\lambda$ in the standard way. Let $J$ be the set of finite subsets of $\lambda$. The family $\{\{X \in J: \beta \in X\}: \beta<\lambda\}$ is a regular family on $J$ and hence gives rise to one on $\lambda$.) We extend $E$ to $\mathscr{D}_{0}$ by closing under finite intersections.

Case 2: $\alpha=2 \beta+1$. We make a choice between $B_{\beta} \in \mathscr{D}_{\alpha}$ and $\left(\lambda \backslash B_{\beta}\right) \in \mathscr{D}_{\alpha}$ so that $\emptyset \notin \operatorname{Fil}\left(\mathscr{D}_{\alpha}\right)$.

Case 3: $\alpha=2 \beta+2$. Let $\left\{C_{l}^{\alpha}: l<\lambda\right\}$ list $\mathscr{D}_{2 \beta+1}$. If there is $S \in[\lambda]^{<\lambda}$ such that $\bigcup_{\epsilon \in S} \epsilon / E_{\beta} \neq \varnothing \bmod \operatorname{Fil}\left(\mathscr{D}_{2 \beta+1}\right)$, we let $\mathscr{D}_{2 \beta+2}=\mathscr{D}_{2 \beta+1}$. So let us assume the following.

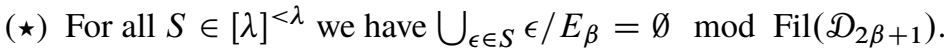

We prove the following auxiliary.

Subclaim There are $\left(\epsilon_{i}, \gamma_{i}\right), i<\lambda$ such that

(a) $\epsilon_{i} \in \lambda \backslash\left\{\epsilon_{j}: j<i\right\}$,

(b) $\gamma_{i} \sim E_{\beta} \epsilon_{i}$

(c) $u_{\beta, \gamma_{i}} \nsupseteq C_{i}^{\alpha} \cap \epsilon_{i} / E_{\beta}$.

Let us first suppose that the subclaim is true and that we have such a sequence $\left(\epsilon_{i}, \gamma_{i}\right), i<\lambda$. Choose $f$ by letting $f\left(\epsilon_{i}\right)=\gamma_{i}$. So $\bigcup_{i \in \lambda / E_{\beta}} u_{\beta, f(i)}$ is a subset of $\lambda$, which includes no element of $\mathscr{D}_{2 \beta+1}$. So we let

$$
\mathscr{D}_{\alpha}=\mathscr{D}_{2 \beta+1} \cup\left\{A \backslash \bigcup_{i \in \lambda / E_{\beta}} u_{\beta, f(i)}: A \in \mathscr{D}_{2 \beta+1}\right\} .
$$

This is clearly closed under finite intersections and does not contain $\emptyset$, and every set in $\mathscr{D}_{\alpha}$ has cardinality $\lambda$.

Let us then prove the subclaim. Let $i<\lambda$, and let

$$
W_{1}=\bigcup_{j<i} \epsilon_{j} / E_{\beta} .
$$

By our assumption $(\star), W_{1}=\emptyset \bmod \operatorname{Fil}\left(D_{2 \beta+1}\right)$. Choose $\xi_{i}$ from the nonempty set $\left(\lambda \backslash W_{1}\right) \cap C_{\alpha, i}$. Then pick $\epsilon_{i}$ so that $\xi_{i} \sim_{E_{\beta}} \epsilon_{i}$. Finally, let

$$
W_{2}=\left\{\gamma<\lambda: \gamma \sim_{E_{\beta}} \epsilon_{i} \text { and } \xi_{i} \in u_{\beta, \gamma}\right\} .
$$

Since $\mathcal{A}^{\beta}$ is a regular family, the set $W_{2}$ is finite. So there is $\gamma_{i} \in u_{\beta, \epsilon_{i}} \backslash W_{2}$. This ends the construction of the sequence $\left(\epsilon_{i}, \gamma_{i}\right), i<\lambda$, and thereby finishes the proof of the subclaim.

Finishing the proof Now that we have constructed the sequence $\mathscr{D}_{\alpha}, \alpha<\lambda^{+}$, we can let

$$
D=\bigcup_{\alpha<\lambda^{+}} \mathscr{D}_{\alpha}
$$

This is an ultrafilter on $\lambda$. It is regular by $(\mathrm{C}-\mathrm{d})$. Now we can easily see that $D$ is not doubly regular. Suppose $E_{\beta}$ and $\bar{u}_{\beta}$ witnesses that $D$ is doubly regular. Let us look at the construction of $\mathscr{D}_{2 \beta+2}$. In the first case we assumed that there is $S \in[\lambda]^{<\lambda}$ with $\bigcup_{\epsilon \in S} \epsilon / E_{\beta} \neq \varnothing \bmod \operatorname{Fil}\left(D_{2 \beta+1}\right)$. So $\bigcup_{\epsilon \in S} \epsilon / E_{\beta} \neq \varnothing \bmod D$, and (DR-b) is violated. In the second case we found $f$ such that $\bigcup_{i \in \lambda / E_{\beta}} u_{\beta, f(i)}=\emptyset$ $\bmod \operatorname{Fil}\left(\mathscr{D}_{\alpha}\right)$. Hence $\bigcup_{i \in \lambda / E_{\beta}} u_{\beta, f(i)}=\emptyset \bmod D$, and (DR-d) is violated. 
Note that double ${ }^{+}$regularity of $D$ implies $\square_{\lambda, D}^{\text {fin }}$ on a regular cardinal $\lambda>\boldsymbol{\aleph}_{0}$ (see Theorem 5), but in light of Theorem 7, not conversely, as GCH implies $\square_{\lambda, D}^{\mathrm{fin}}$ for regular $D$ and regular $\lambda$ (see [7, Lemma 4]).

Theorem 7 has the assumption that $2^{\lambda}=\lambda^{+}$, which may fail for all $\lambda$. We present next a slightly different construction under a different assumption, one that is always satisfied by a multitude of cardinals $\lambda$.

Theorem 8 Assume the following two conditions.

(A1) $\operatorname{cof}(\lambda)>\aleph_{0}$ or $\lambda>2^{\boldsymbol{\aleph}_{0}}$.

(A2) There is $\mathcal{A} \subseteq \mathscr{P}(\lambda)$ of cardinality $2^{\lambda}$ such that $|\{A \cap i: A \in \mathcal{A}\}| \leq \lambda$ for all $i<\lambda$.

Then there is a regular but not doubly regular filter on $\lambda$.

Note that a family $\mathcal{A}$, as in (A2), always exists if $\lambda=2^{<\lambda}$. Hence condition (A2) can be replaced by $\lambda=\beth_{\alpha}, \alpha$ limit.

Proof Let $\left\langle\left(E_{\beta}, \bar{u}_{\beta}\right): \beta<2^{\lambda}\right\rangle$ list all pairs where $E_{\beta}$ is an equivalence relation on $\lambda$ and $\bar{u}_{\beta}^{i}=\left\langle u_{\beta, \epsilon}: \epsilon \sim E_{\beta} i\right\rangle$ is a regular family of subsets of $i / E_{\beta}$ for each $i<\lambda$. Let $\left\{B_{\alpha}: \alpha<2^{\lambda}\right\}$ list $\mathcal{P}(\lambda)$.

We construct a sequence $\left(I_{\alpha}, \mathscr{D}_{\alpha}\right), \alpha<2^{\lambda}$ such that

1. $\left|I_{\alpha}\right| \leq|\alpha|, I_{\alpha} \subseteq \mathcal{P}(\lambda),\left(I_{\alpha}\right)$ is continuously increasing;

2. $\mathscr{D}_{\alpha}$ is the filter $\mathscr{D}\left[I_{\alpha}\right]=\left\{A \subseteq \lambda: \exists J \in\left[I_{\alpha}\right]^{<\aleph_{0}} \exists S \in[\lambda]^{<\lambda}(\bigcap J \subseteq A \cup S)\right\}$;

3. $\mathscr{D}_{2 \beta+1}=\mathscr{D}_{2 \beta} \cup\left\{B_{\beta}\right\}$ or $\mathscr{D}_{2 \beta+1}=\mathscr{D}_{2 \beta} \cup\left\{\lambda \backslash B_{\beta}\right\}$;

4. $\mathscr{D}_{2 \beta+2}$ satisfies:

(a) there is some $W \in[\lambda]^{<\lambda}$ such that $\bigcup_{i \in W} i / E_{\beta} \neq \emptyset \bmod \mathscr{D}_{2 \beta+1}$, or

(b) there is an $f$ such that $f\left(i / E_{\beta}\right) \in i / E_{\beta}$ for all $i$ and $\lambda \backslash \bigcup\left\{u_{\beta, f(x)}\right.$ : $\left.x \in \lambda / E_{\beta}\right\} \in I_{\beta}$, or

(c) $\left|\left\{X \in \lambda / E_{\beta}:|X \cap B|=\lambda\right\}\right|<\lambda$ for some $B \in D_{2 \beta+1}$.

The construction now follows. Let us look at the case $\alpha=2 \beta+2$. If we cannot form $\mathscr{D}_{\alpha}$ as required, then

(N1) if $W \in[\lambda]^{<\lambda}$, then $\bigcup_{i \in W} i / E_{\beta}=\emptyset \bmod \mathscr{D}_{2 \beta+1}$;

(N2) if $f$ is a function such that $\operatorname{dom}(f)=\lambda / E_{\beta}$ and $f\left(i / E_{\beta}\right) \sim_{E_{\beta}} i$ for all $i<\lambda$, and

$$
A_{\beta, f}=\bigcup\left\{u_{\beta, f(x)}: x \in \lambda / E_{\beta}\right\},
$$

then $\emptyset \in \mathscr{D}\left(I_{2 \beta+1} \cup\left\{\lambda \backslash A_{\beta, f}\right\}\right)$;

(N3) for $B \in \mathscr{D}_{2 \beta+1},\left|\left\{X \in \lambda / E_{\beta}:|X \cap B|=\lambda\right\}\right|=\lambda$.

We derive a contradiction. This will ensure that $\mathcal{D}_{\alpha}$ can be found. Let $\left\langle x_{\beta, i}: i<\lambda\right\rangle$ list $\lambda / E_{\beta}$. By our choice of $\mathcal{A}$, there are one-one functions $b_{i}:\{A \cap i: A \in \mathcal{A}\} \rightarrow x_{\beta, i}$ for each $i<\lambda$. If $s \subseteq \lambda$, let $g_{s}$ be a function such that $\operatorname{dom}\left(g_{s}\right)=\lambda / E_{\beta}$ and

$$
g_{s}\left(x_{\beta, i}\right)=b_{i}(s \cap i)
$$

so that $g_{s}\left(x_{\beta, i}\right) \in x_{\beta, i}$. By (N2) there are

$$
J_{\beta, s} \in\left[I_{2 \beta+1}\right]^{<\aleph_{0}}, \quad W_{\beta, s} \in[\lambda]^{<\lambda}
$$

such that

$$
\bigcap_{B \in J_{\beta, s}} B \subseteq A_{\beta, g_{s}} \cup W_{\beta, s} .
$$


Since $|\mathcal{A}|=2^{\lambda}$, there are $J_{*} \in\left[I_{2 \beta+1}\right]^{<\aleph_{0}}$ and $\mu<\lambda$ such that if

$$
\mathcal{A}_{1}=\left\{s \in \mathcal{A}: J_{\beta, s}=J_{*},\left|W_{\beta, s}\right|=\mu\right\},
$$

then $\left|\mathcal{A}_{1}\right|=2^{\lambda}$. Let $B_{*}=\bigcap J_{*} \in \mathscr{D}_{2 \beta+1}$. By (N3),

$$
\left|\left\{j<\lambda:\left|x_{\beta, j} \cap B_{*}\right|=\lambda\right\}\right|=\lambda \text {. }
$$

Claim There are $s_{n} \in \mathcal{A}_{1}, n<\omega$, and $i<\omega$ such that $s_{n} \cap i \neq s_{m} \cap i$ for all $n<m<\omega$.

Case 1: $\operatorname{cof}(\lambda)>\boldsymbol{\aleph}_{0}$. Pick distinct $s_{n} \in \mathcal{A}_{1}, n<\omega$. Since $\operatorname{cof}(\lambda)>\boldsymbol{\aleph}_{0}$, there is $i<\lambda$ such that $s_{n} \cap i \neq s_{m} \cap i$ for all $n<m<\omega$.

Case 2: $\operatorname{cof}(\lambda)=\aleph_{0}, \lambda>2^{\aleph_{0}}$. Pick distinct $s_{\xi} \in \mathcal{A}_{1}, \xi<\left(2^{\aleph_{0}}\right)^{+}$. Let $C \subseteq \lambda$ be cofinal, $|C|=\boldsymbol{\aleph}_{0}$. Let $\chi:\left[\left(2^{\aleph_{0}}\right)^{+}\right]^{2} \rightarrow C$ be defined by $\chi(\{\xi, \zeta\})=\min \{c \in C$ : $\left.s_{\xi} \cap c \neq s_{\zeta} \cap c\right\}$. By the Erdôs-Rado theorem $\left(2^{\aleph_{0}}\right)^{+} \rightarrow\left(\boldsymbol{\aleph}_{1}\right)_{\aleph_{0}}^{2}$, there is $i \in C$ and an uncountable $H \subseteq\left(2^{\aleph_{0}}\right)^{+}$such that $\chi \uparrow[H]^{2}$ has constant value $i$.

The claim is proved. By (2), there is $j>i$ such that $\left|B_{*} \cap x_{\beta, j}\right|=\lambda$. With the notation of (N2),

$$
A_{\beta, g_{s_{n}}} \cap x_{\beta, j}=u_{\beta, b_{j}\left(s_{n} \cap j\right)}
$$

and the sets $u_{\beta, b_{j}\left(s_{n} \cap j\right)}$ are distinct because $b_{j}$ is one-one. By regularity,

$$
\bigcap_{n} u_{\beta, b_{j}\left(s_{n} \cap j\right)}=\emptyset \text {. }
$$

Let $W=\bigcup\left\{W_{\beta, s_{n}}: n<\omega\right\}$. Clearly, $|W|=\mu$. Now

$$
B_{*} \cap x_{\beta, j} \subseteq u_{\beta, b_{j}\left(s_{n} \cap j\right)} \cup W .
$$

This contradicts $\left|B_{*} \cap x_{\beta, j}\right|=\lambda$, since $|W|=\mu$ and (3) gives

$$
B_{*} \cap x_{\beta, j} \subseteq \bigcap_{n}\left(u_{\beta, b_{j}\left(s_{n} \cap j\right)} \cup W\right)=W .
$$

If we start with a model of $\mathrm{GCH}$, we can use Easton forcing (see [3]) to obtain a model in which $2^{\lambda}$ is-for all regular $\lambda$-anything not ruled out by the conditions $\kappa \leq \lambda \Rightarrow 2^{\kappa} \leq 2^{\lambda}$ and $\operatorname{cof}\left(2^{\lambda}\right)>\lambda$. In the arising forcing extension $V[G]$ the tree $(<\bar{\lambda} 2)^{V}, \lambda$ regular, has cardinality $\lambda$ and $2^{\lambda}$ branches. Hence we have in $V[G]$ a set $\mathcal{A}_{\lambda}$ of cardinality $2^{\lambda}$-for all regular $\lambda$ - such that $\forall i<\lambda\left(\left|\left\{A \cap i: A \in \mathcal{A}_{\lambda}\right\}\right| \leq \lambda\right)$, which is exactly the assumption (A2) of Theorem 8 .

\section{Good Ultrafilters}

Keisler [6] introduced the concept of $\kappa$-goodness of ultrafilters and proved that if $2^{\lambda}=\lambda^{+}$and if $D$ is a $\lambda^{+}$-good (i.e., good) countably incomplete ultrafilter on $\lambda$, then $\prod_{D} \mathcal{M}_{i} \cong \prod_{D} \mathcal{N}_{i}$ for any models $\mathcal{M}_{i} \equiv \mathcal{N}_{i}$ of cardinality at most $\lambda^{+}$in a vocabulary of cardinality at most $\lambda$. This raises the question whether there is a connection between goodness and double regularity. It turns out that these concepts are independent of each other.

Proposition 9 Suppose that $\lambda>\boldsymbol{\aleph}_{0}$. There is a doubly regular ultrafilter on $\lambda$ which is not good. If $2^{\lambda}=\lambda^{+}$, then there is a good countably incomplete ultrafilter on $\lambda$ which is not doubly regular. 
Proof For the first claim, let $D_{1}$ be a doubly regular ultrafilter on $\lambda$ (exists by Observation 4), and let $D_{2}$ be a countably incomplete ultrafilter of $\omega$ which is not $\aleph_{2}$-good (exists by [6, Theorem 5.1]). Let $D=D_{1} \times D_{2}$. This is an ultrafilter on the set $\lambda \times \omega$ of size $\lambda$. Since $D_{2}$ is not $\lambda^{+}$-good, neither is $D$ (see [13, Chapter VI, Lemma 3.7]). Double regularity is inherited from $D_{1}$ as follows. Suppose that we have pairwise disjoint sets $u_{i}, i<\lambda$, on $\lambda$, each of cardinality $\lambda$, and regular filters $F_{i}$ on $u_{i}$ such that for all $A \subseteq \lambda$ :

$$
\left[\forall^{\infty} i<\lambda\left(A \cap u_{i} \in F_{i}\right)\right] \rightarrow A \in D_{1} .
$$

Let $G_{i} \subseteq F_{i}$ be a regular family on $u_{i}$. Let $u_{i}^{*}=u_{i} \times \omega$ and $G_{i}^{*}=\left\{A \times \omega: A \in G_{i}\right\}$. Let $F_{i}^{*}$ be the filter on $u_{i}^{*}$ generated by $\left\{A \times \omega: A \in F_{i}\right\}$. Now $G_{i}^{*}$ is a regular family $\subseteq F_{i}^{*}$, and if $A \subseteq \lambda \times \omega$, then

$$
\left[\forall^{\infty} i<\lambda\left(A \cap u_{i}^{*} \in F_{i}^{*}\right)\right] \rightarrow A \in D_{1} \times D_{2} .
$$

This ends the proof that $D$ is doubly regular.

For the second claim we use a combination of the construction of the proof of Theorem 7 and Keisler's construction of a good ultrafilter in [6, Theorem 4.4]. The construction of Keisler as presented in [1, Chapter 6, p. 387] proceeds in stages, generating a continuously increasing sequence $F_{\alpha}, \alpha<2^{\lambda}$, of filters such that the following condition holds (for unexplained terminology we refer to [1, Chapter 6, p. 387]). For the first (in a fixed well-ordering) monotone $f:[\lambda]^{<\boldsymbol{\aleph}_{0}} \rightarrow F_{\alpha}$ for which there is no additive extension $[\lambda]^{<\aleph_{0}} \rightarrow F_{\alpha}$, there is an additive extension $g:[\lambda]^{<\aleph_{0}} \rightarrow F_{\alpha+1}$. To make sure that such $g$ and $F_{\alpha+1}$ always exist, an auxiliary sequence is simultaneously defined, namely, a descending sequence $\Pi_{\alpha}, \alpha<2^{\lambda}$, of partitions of $\lambda$, starting from a carefully chosen initial set $\Pi_{0}$ with $\left|\Pi_{0}\right|=2^{\lambda}$. There is no problem in interleaving the inductive construction of the filters $F_{\alpha}$ into the construction in the proof of Theorem 7 . The resulting ultrafilter is good but not doubly regular.

\section{Concluding Remarks}

We proved that $\square_{\lambda, D}^{\text {fin }}$ holds if $\lambda$ is a regular cardinal and $D$ is a doubly regular filter. This naturally raises the question whether $\square_{\lambda, D}^{\text {fin }}$ can fail at a regular cardinal for some regular, but not doubly regular, filter. We know that it can fail at a singular cardinal (see [8]).

Conjecture 1 Consistently, $\square_{\lambda, D}^{\text {fin }}$ fails for some regular $\lambda>\omega$ and some regular filter $\lambda$ generated by $\lambda$ sets.

Conjecture 2 If $D$ is a regular ultrafilter on $\aleph_{1}$ such that $\neg \square{ }_{\aleph_{1}, D}^{\text {fin }}$, then for any increasing continuous $\left\langle\alpha_{i}: i<\omega_{1}\right\rangle$ with $\alpha_{i}<\omega_{1}$, there is $A \in D$ such that $A \cap\left[\alpha_{i}, \alpha_{i+1}\right)$ is finite for all $i<\omega_{1}$.

Note that if

$$
D=\left\{A \subseteq \omega_{1}: \forall^{\infty} i<\lambda\left(A \cap\left[\alpha_{i}, \alpha_{i+1}\right) \in D_{i}\right)\right\},
$$

$D_{i}$ ultrafilter on $\left[\alpha_{i}, \alpha_{i+1}\right)$, then the answer to Conjecture 2 is positive. This may indicate that looking for counterexamples for $\square_{\aleph_{1}, D}^{\mathrm{fin}}$ can be hard. 


\section{Note}

1. The usual elementary equivalence in a finite relational vocabulary is thus $E F_{n}$ equivalence for all $n<\omega$, and $L_{\infty \omega}$-equivalence is the same as $E F_{\omega}$-equivalence. For models of cardinality at most $\kappa, E F_{\kappa}$-equivalence is equivalent to isomorphism.

\section{References}

[1] Chang, C. C., and J. Keisler, Model Theory, 3rd edition, North-Holland, Amsterdam, 1990. 418, 426

[2] Deiser, O., and D. Donder, "Canonical functions, non-regular ultrafilters and Ulam's problem on $\omega_{1}$," Journal of Symbolic Logic, vol. 68 (2003), pp. 713-39. MR 2000073. DOI 10.2178/js1/1058448434. 422

[3] Easton, W. B., "Powers of regular cardinals," Annals of Mathematical Logic, vol. 1 (1970), pp. 139-78. Zbl 0209.30601. MR 0269497. 425

[4] Hyttinen, T., “On $\kappa$-complete reduced products," Archive for Mathematical Logic, vol. 31 (1992), pp. 193-99. MR 1147741. DOI 10.1007/BF01269947. 418

[5] Jech, T., Set Theory, 3rd edition, Springer, Berlin, 2003. 420

[6] Keisler, H. J., "Good ideals in fields of sets," Annals of Mathematics (2), vol. 79 (1964), pp. 338-59. Zbl 0137.00803. MR 0166105. 425, 426

[7] Kennedy, J., and S. Shelah, "On regular reduced products," Journal of Symbolic Logic, vol. 67 (2002), pp. 1169-77. Zbl 1012.03048. MR 1926605. DOI 10.2178/js1/1190150156. 417, 418, 419, 424

[8] Kennedy, J., and S. Shelah, "More on regular reduced products," Journal of Symbolic Logic, vol. 69 (2004), pp. 1261-66. Zbl 1071.03033. MR 2135667. DOI 10.2178/js1/1102022222. 417, 418, 426

[9] Kennedy, J., S. Shelah, and J. Väänänen, "Regular ultrafilters and finite square principles," Journal of Symbolic Logic, vol. 73 (2008), pp. 817-23. Zbl 1160.03026. MR 2444269. DOI 10.2178/js1/1230396748. 418

[10] Ketonen, J., "Nonregular ultrafilters and large cardinals," Transactions of the American Mathematical Society, vol. 224 (1976), pp. 61-73. Zbl 0352.02045. MR 0419236. 422

[11] Prikry, K., "On a problem of Gillman and Keisler," Annals of Mathematical Logic, vol. 2 (1970), pp. 179-87. Zbl 0209.30701. MR 0269513. 422

[12] Shelah, S., "'Gap 1' two-cardinal principles and the omitting types theorem for L(Q)," Israel Journal of Mathematics, vol. 65 (1989), pp. 133-52. Zbl 0681.03016. MR 0998667. DOI 10.1007/BF02764857. 418

[13] Shelah, S., Classification Theory and the Number of Nonisomorphic Models, 2nd ed., vol. 92 of Studies in Logic and the Foundations of Mathematics, North-Holland, Amsterdam, 1990. MR 1083551. 422, 426

[14] Shelah, S., "The combinatorics of reasonable ultrafilters," Fundamenta Mathematicae, vol. 192 (2006), pp. 1-23. Zbl 1115.03058. MR 2283626. DOI 10.4064/fm192-1-1. 421

\section{Acknowledgments}

The first and third authors' work was partially supported by Academy of Finland grant 251557. The second author would like to thank the Israel Science Foundation for partial support of this research through grant no. 1053/11, Publication 1011. 
Kennedy

Department of Mathematics and Statistics

00014 University of Helsinki

Finland

and

Helsinki Collegium for Advanced Studies

Fabianinkatu 24 (P.O. Box 4)

00014 University of Helsinki

Finland

Shelah

Institute of Mathematics

Hebrew University

Jerusalem

Israel

and

Department of Mathematics

Rutgers University

Hill Center-Busch Campus

Piscataway, New Jersey 08854-8019

USA

\section{Väänänen}

Department of Mathematics and Statistics

00014 University of Helsinki

Finland

and

Institute for Logic, Language and Computation

University of Amsterdam

1090 GE Amsterdam

The Netherlands 